\title{
Erratum to: Isolated Calyx Mistaken for a Cyst: Inappropriately Performed Catheter-Directed Sclerotherapy and Safe Removal of the Catheter After Selective Embolization
}

JungWon Kwak · Seung Hwa Lee $\cdot$ Hwan Hoon Chung •

Bo Kyung Je $\cdot$ Suk kyu Yeom $\cdot$ Deuk Jae Sung

Published online: 6 December 2014

(C) Springer Science+Business Media New York and the Cardiovascular and Interventional Radiological Society of Europe (CIRSE) 2014

Erratum to: Cardiovasc Intervent Radiol

DOI 10.1007/s00270-014-0871-8

The first author's name was misspelled in the published article. "Jng Won Gwak" should be "JungWon Kwak" as shown in this erratum.

The online version of the original article can be found under doi:10.1007/s00270-014-0871-8.

J. Kwak · S. H. Lee $(\varangle) \cdot$ H. H. Chung · B. K. Je · S. k. Yeom

Department of Radiology, Ansan Hospital, Korea University

College of Medicine, 516 Gojan-dong Danwon-gu, Ansan-si,

Gyeonggi-do 425-707, Korea

e-mail: gareureung@daum.net

J. Kwak

e-mail: jjungwonie@hanmail.net

H. H. Chung

e-mail: chungmic@korea.ac.kr

B. K. Je

e-mail: purity21@hanmail.net

S. k. Yeom

e-mail: pagoda20@hanmail.net

D. J. Sung

Department of Radiology, Anam Hospital, Korea University

College of Medicine, Anam-dong 5ga, Seongbuk-gu 136-705,

Korea

e-mail: urora@korea.ac.kr 